\title{
Proof-Carrying Plans
}

\author{
Christopher Schwaab ${ }^{1}$, Ekaterina Komendantskaya ${ }^{2}$, Alasdair Hill ${ }^{2}$, \\ František Farka ${ }^{1,2}$, Ronald P. A. Petrick ${ }^{2}$, Joe Wells ${ }^{2}$, and Kevin Hammond ${ }^{1}$ \\ 1 School of Computer Science, University of St Andrews, UK \\ \{cjs26,ff32, kh8\} est-andrews.ac.uk \\ 2 Department of Computer Science, Heriot-Watt University, UK \\ $\{$ ek19, ath7, rpp6, jbw $@$ @hw.ac.uk
}

\begin{abstract}
As AI applications are deployed in more and more real-world settings, it is becoming increasingly important to verify their safety and security. While declarative languages (of the kind found in automated planners and model checkers) are traditionally used for verifying AI systems, a big challenge is to design methods that generate verified executable programs. A good example of such a "verification to implementation" cycle is given by automated planning languages like PDDL, where plans are found via a model search in a declarative language, but then interpreted or compiled into executable code in an imperative language. In this paper, we show that this method can itself be verified. We present a formal framework and a prototype Agda implementation that represent PDDL plans as executable functions that inhabit types that are given by formulae describing planning problems. By exploiting the well-known Curry-Howard correspondence, type-checking then automatically ensures that the generated program corresponds precisely to the specification of the planning problem.
\end{abstract}

Keywords: AI Planning · Curry-Howard Correspondence · Constructive Logic · Verification · Dependent Types.

\section{Motivation}

Declarative programming languages have long provided convenient formalisms for knowledge representation and reasoning, ranging from Lisp and Prolog in the 1960s1980s to modern SMT solvers [3,2], model checkers [13], and automated planners $[4,11]$. Common features of such languages typically include a clear logic-based syntax, a well-understood declarative semantics, and an inference engine that produces sound results with respect to the semantics.

As AI applications become increasingly deployed in the real world, e.g. in selfdriving vehicles or autonomous robots, so safety and security issues are becoming increasingly important. Existing ad-hoc software development approaches do not provide the strong confidence levels that the public expects from such applications. It is tempting to envisage that declarative languages will play an increasingly important role in verifying the safety and security of real-world AI applications. Ideally, such languages could become vehicles for proof-carrying code, an approach in which all relevant verification properties are directly embedded in the source code [12]. To make this possible, 
we must supplement the ability to prove that a property $A$ holds in a theory $T$ (denoted $T \vdash A$ ) with robust mechanisms that can generate a program $p$ that executes according to the specification $A$, together with a proof that $p$ satisfies $A$ (denoted $T \vdash p: A$ ). Such an approach would embed verification directly as an integral part of the implementation cycle for AI applications.

The well-known Curry-Howard correspondence [18,19], tells us, of course, that some proofs in intuitionistic first-order logic can be represented as computable functions. In this case, first-order formulae are seen as types, and proofs are seen as terms that inhabit those types. For example, when we write $T \vdash p: A$, we mean that a proof term $p$ is an executable program that satisfies proposition $A$, and moreover that this inference is sound, i.e. that $T \vdash p: A$ holds in some formal system.

Until recently, the significance of the Curry-Howard correspondence has been predominantly theoretical. The main impediment to its practical application has been the immaturity of programming languages that could fully implement this idea. For example, in order to express verification properties of AI applications, a language that could infer $T \vdash p: A$ must possess at least first-order types. Moreover, it should ideally also possess dependent types. Several dependently-typed languages have now become available and increasingly practical, e.g., Coq, Agda, and Idris. This development has made it possible to re-open the discussion of the actual practical value of the CurryHoward correspondence. For example, in [6,7] Fu et al. have given a Curry-Howard interpretation for first-order Horn clauses and the resolution algorithm; and Urzyczyn and Schubert [16] have given a constructive semantics for answer set programming (ASP).

In this paper, we turn our attention to AI Planning languages [4,11] - a rapidly growing research and engineering area that develops methods and tools for generating plans from declarative problem specifications. We show that the Planning Domain Definition Language (PDDL) [11] is a natural domain for the Curry-Howard implementation of declarative reasoning. In particular, specifications of planning problems that are usually written in first-order logic can be expressed naturally as types, and executable plans that are generated by PDDL can be formalised as programs that inhabit those types. Type checking thus verifies that correct executable programs are generated from specifications via the automated planning tool. We provide a proof-of-concept implementation [17] in the dependently-typed language Agda.

\section{Example: Proof-Carrying PDDL}

Figure 1 shows a snippet of PDDL code that describes the classic Blocksworld domain, a simple planning task for a robot assembling a tower from bricks on a table. It defines a set of predicates (handEmpty, holding, onTable, on, clear) and an action pickup_from_table that must satisfy certain pre- and post-conditions ("effects") that are expressed using those predicates. Several such actions are usually defined as part of a planning domain. In addition, a grounded problem will also be supplied to the planner, e.g., to form a stack of blocks $a$ on $b$ on $c$, given that $a, b$ and $c$ are initially on the table (but not on each other). Given the domain and problem definitions, an automated planner will initiate an algorithm (e.g., a search procedure) to generate a sequence of actions that satisfy the specification and the goal. In our case, one possible solution is: 


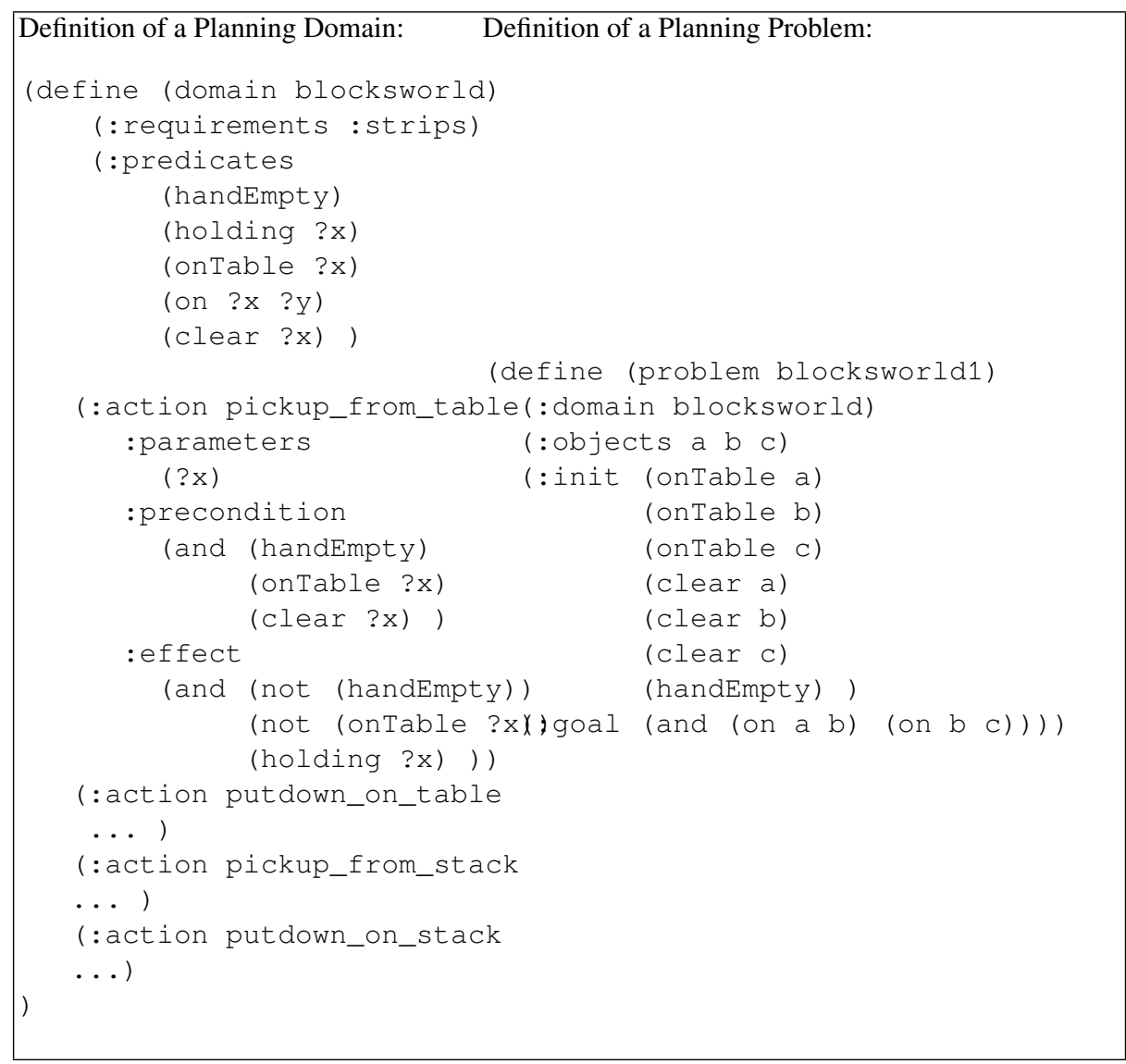

Fig. 1. The Blocksworld: a code snippet defining the planning domain and a planning problem.

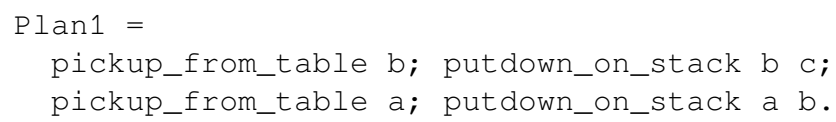

We would like to have an implementation of this planning language where an executable function $\mathrm{plan}_{1}$ is generated from the planning domain and problem, such that $\mathrm{plan}_{1}$ corresponds to the actions of Plan1 and has a type onTable a $\wedge$ onTable $b \wedge$

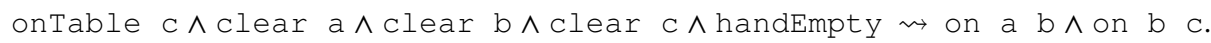
If this judgement type-checks, then we will obtain a verified program plan $_{1}$ that can be later compiled and executed. As we will show in the rest of the paper, this task is far from trivial. Although the Curry-Howard correspondence tells us that, in principle, (intuitionistic) first-order proofs have a computational meaning, it is not enough for us to just formulate arbitrary proofs. Firstly, we need to formulate a generic and automatable approach to translate PDDL domains and problems into the dependently-typed setting. In addition, we need to devise our language translation and calculus in such a way as 


$$
\begin{array}{rcll}
\text { Atomic Formulae } & A t \ni A & ::=R C_{1} \ldots C_{n} \\
\text { Formulae } & \text { Form } \ni P, Q & ::=A|\neg A| P \wedge Q \\
\text { Polarities } & \text { Polarity } \ni t & ::=+\mid- \\
\text { States } & \mathcal{N} \ni M, N & ::=[] \mid\left[A^{t}, N\right] \\
\text { Plans } & \mathcal{P l a n} \ni f & ::=\text { halt } \mid \alpha ; f \\
\text { Contexts } & \Gamma \ni \Gamma_{1}, \Gamma_{2} & ::=\alpha: M \rightsquigarrow N
\end{array}
$$

Fig. 2. Definitions of Formulae, States, Plans and Actions, given a set of predicates $\mathcal{R}=$ $\left\{R, R_{1}, R_{2}, \ldots, R_{n}\right\}$, a set of constants $\mathcal{C}=\left\{C_{1}, C_{2}, \ldots, C_{k}\right\}$, and a set of constant actions $\left\{\alpha, \alpha_{1}, \ldots, \alpha_{m}\right\} \cup$ halt.

to ensure that the programs that inhabit the types give us the actual executable plans in the PDDL sense, rather than some arbitrary proof-terms. In this paper, we therefore develop two parallel narratives. The first sets up the general method in mathematical notation independently of the concrete implementation. The second illustrates the important engineering aspect of this work, with reference to the intricacies of the Agda encoding that we give in [17]. The two parallel story lines merge when we come to the main result of this paper: the formal proof of soundness of the proof terms that implement the plans. We state this in standard mathematical notation, but delegate the proof checking to Agda. In time, we envisage that our Agda prototype will become a fully fledged program for generation of executable code from planners, while maintaining the guarantees of soundness of the generated code relative to the plan specification.

\section{Planning Problems as Types}

In their development of the STRIPS planner, Fikes and Nilsson presented an inference system for planning languages that is based on the notion of states, or possible worlds. The worlds are sets of atomic formulae, that interpret complex formula of the planning domain. Operators that are defined on the worlds interpret planning actions, and rewrite the worlds by adding and deleting the atomic formulae. The inference algorithm thus starts in an initial world and ends in a goal world by the repeated application of the operators. The system is sound in the sense that the resultant world model satisfies the goal. We now provide a show how to work with STRIPS predicates directly in a typesystem, yielding proof obligations that will be fulfilled by plan execution.

\subsection{Formal Language and its Declarative Semantics}

We assume a finite set of predicates $\mathcal{R}=\left\{R, R_{1}, R_{2}, \ldots, R_{n}\right\}$ each $R_{i}$ of fixed arity, and a finite set $\mathcal{C}=\left\{C_{1}, C_{2}, \ldots, C_{k}\right\}$ of constants (also known as "objects"). The standard definition of first-order formulae is given in Figure 2. It has two notable restrictions: the formulae do not admit variables, and only atomic formulae can be negated. The former restriction, together with the assumption that there are only finitely many constants, ensures that the set of all atomic formulae is finite, which makes it possible to take the 

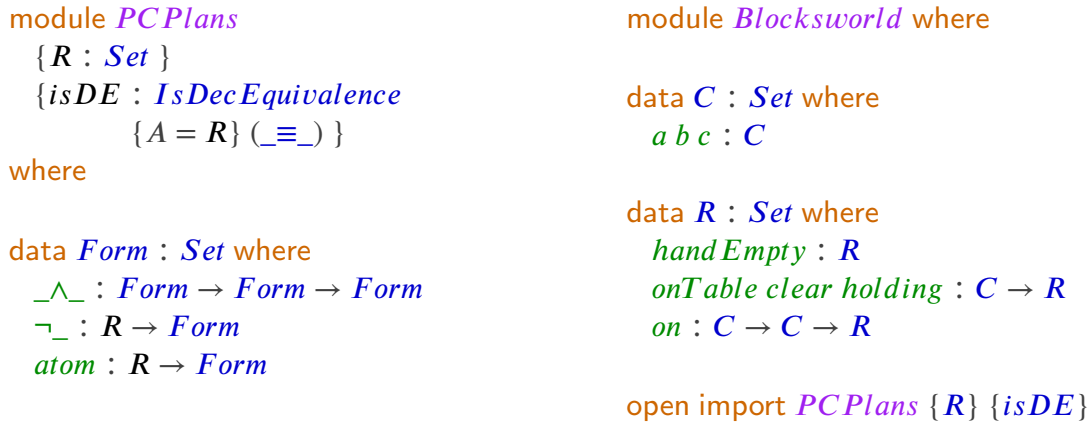

Fig. 3. Left: Module PC Plans giving a general-case Agda definition of a formula, following the set-up of Figure 2. Right: Module Blocksworld giving specification of the particular Blocksworld domain from Figure 1: listing its constants and predicates.

$$
\begin{array}{lll}
\text { World }: \text { Set } & \text { data Polarity }: \text { Set where } & \text { neg : Polarity } \rightarrow \text { Polarity } \\
\text { World }=\text { List } R & +-: \text { Polarity } & n e g+=- \\
& & n e g-=+
\end{array}
$$

Fig. 4. Agda definitions of worlds as lists of atomic formulae, polarities. (Module PC Plans)

closed world assumption [15], and ensure the decidability of set membership on possible worlds. The latter restriction means that disjunction is not definable in our language. In PDDL, two key restrictions apply to the use of disjunction. Firstly, all formulae are pre-compiled into disjunctive normal form. Secondly, no "actions" can have disjunctive "effects", i.e. they cannot give rise to disjunctive post-conditions. Thus, our second restriction actually adheres to the practice of PDDL plan specification and search.

Example 1. Given the syntax of Section 2, handEmpty $\wedge \neg$ $\neg$ Table a is a formula.

The inductive definitions of Figure 2 are given as data type definitions in our Agda implementation (Figure 3). We provide a generic Agda module, PC Plans, that is parametric in predicates and actions. For each planning problem, the set of predicates $\mathcal{R}$ may then be defined concretely, as in the Blocksworld module. Propositional equality on atomic formulae must be shown to be decidable for the particular planning problem. As we will show later, this property is needed in order to manipulate world representation. Since our implementation of the PC Plans module takes a generic approach, a proof that propositional equality $\equiv_{-}$for $\mathcal{R}$ is decidable must also be provided as a module parameter. This explains the declaration of the main module:

$$
\text { module PCPlans }\{\mathcal{R}: \text { Set }\}\left\{\text { is DE : Is DecEquivalence }\{A=\mathcal{R}\}\left({ }_{-}\right)\right\}
$$

When we instantiate $\mathcal{R}$ with a finite set of predicates for each planning problem, we need to instantiate $i s D E$ with a proof that propositional equality for this particular problem is indeed decidable ([17] contains examples of such "boilerplate" proofs).

Given a set $w$ of atomic formulae (called a world), a formula $F$ is satisfied by $w$ if $w k_{t} F$ (where $t \in\{+,-\}$ ) can be derived using the rules of Figure 5. In Agda, we 


$$
\frac{w k_{t} P \quad w k_{t} Q}{w k_{t} P \wedge Q} \frac{w k_{-t} P}{w k_{t} \neg P} \frac{A \in w}{w k_{+} A} \frac{A \notin w}{w k_{-} A}
$$

Fig. 5. Declarative interpretation of formulae. We define $-t$ by taking $-+=-$ and $--=+$.

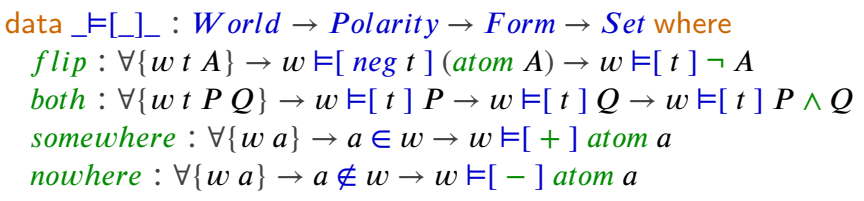

Fig. 6. Agda definition of the entailment relation given in Figure 5. (Module PCPlans)

take advantage of the extensive library of list operations, and so define worlds as lists of atomic formulae, as shown in Figure 4. Figure 7 gives an Agda definition of the entailment relation.

Example 2. Given the world $w_{1}=\{$ handEmpty $\}$, we have $w_{1} k_{+}$handEmpty $\left.\wedge\right\urcorner$ onTable a.

It might be expected that the rule for conjunction with negative polarity to be given by two additional rules: $\frac{w k_{-} P}{w k_{-} P \wedge Q}$ and $\frac{w k_{-} Q}{w k_{-} P \wedge Q}$. However, our current rule is sound given the syntax restrictions, and it simplifies our reasoning on decidability of normalisation, which we define next.

\subsection{Operational Semantics, States and Types}

Matching the declarative-style semantics of Figure 5, we can define an operational semantics, given by a normalisation function that acts directly on formulae and computes lists of atomic formulae with polarities. A state is defined as a list of atomic formulae with polarities, as in Figure 2. By a small abuse of notation, we will use $\in$ to denote list membership, as well as set membership. The function $\downarrow_{t}$ normalises a formula to a state:

$$
\begin{aligned}
(P \wedge Q) \downarrow_{t} N & =Q \downarrow_{t} P \downarrow_{t} N \\
\neg A \downarrow_{t} N & =A \downarrow_{-t} N \\
A \downarrow_{t} N & =\left[A^{t}, N\right]
\end{aligned}
$$

We write $P \downarrow_{t}$ to mean $P \downarrow_{t}[$ ].

Example 3. Continuing with the previous examples, we have:

(handEmpty $\wedge$ ᄀonTable a) $\downarrow_{+}=\left[\right.$handEmpty $^{+}$, onTable a $\left.{ }^{-}\right]$.

As might be expected, while the definition of the entailment relation $k_{t}$ is given as an inductive data type in Agda, the normalisation is defined as a function (Figure 7). Note again that, in order to bring the disjunction into this language in any future extensions, 


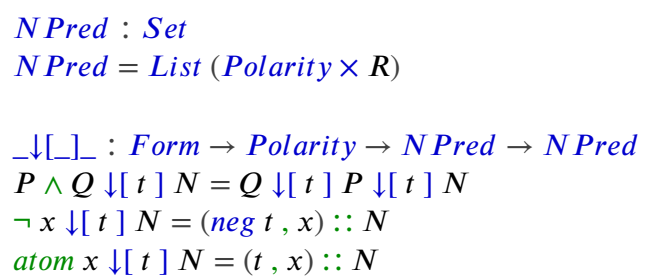

Fig. 7. Agda definition of the normalisation function. (Module PCPlans)

normalisation function for minus could be amended, to allow for non-determinism. Normalisation is sound relative to the declarative interpretation of formulae. Given a state $N$, define a well-formed world $w_{N}$ to contain all $A$ such that $A^{+} \in N$ and contain no $A$ 's such that $A^{-} \notin N$. Generally $w_{N}$ is not uniquely defined, and we use the notation $\left\{w_{N}\right\}$ to refer to the (necessarily finite) set of all $w_{N}$. We then have the result:

Theorem 1 (Soundness and completeness of normalisation). Given a formula $P$ and a world $w$, it holds that $w k_{t} P$ iff $w \in\left\{w_{P \downarrow_{t}}\right\}$.

Proof. $(\Rightarrow)$ is proven by induction on the derivation of $w k_{t} P .(\Leftarrow)$ follows by induction on the shape of $P$, cf. the attached Agda file [17] for the fully formalised proof.

Example 4. If $N=$ (handEmpty $\wedge$ ᄀonTable a) $\downarrow_{+}$, then $w_{N}$ may be given by e.g. $w_{1}=\{$ handEmpty $\}$, or $w_{2}=\{$ handEmpty, onTable $\mathrm{b}\}$, or any other world containing handEmpty but not onTable a. The given formula will be satisfied by any such $w_{N}$.

Theorem 1 will allow us to work with states at the type level, while keeping the link to the standard PDDL formula syntax and declarative semantics.

We finally define actions and plans. Given a halting state halt, and a finite set of constant actions $\left\{\alpha, \alpha_{1}, \ldots, \alpha_{m}\right\}$, we define plans inductively as sequences of action names ending with halt, cf. Figure 2. Once again, we show an instantiated version of the Agda definition of actions in Figure 8, with actions specified as per the Blocksworld problem. In the Agda prototype [17], we first develop the code for an abstract set Action, and then instantiate it on the concrete examples. Figure 8 also shows the Agda function plan $_{1}$ that encodes Plan1 given in Section 2 in PDDL syntax. Keeping in line with Section 2 , a planning domain (or a context) $\Gamma$ is a set of actions with effects, of the form $\alpha: N \rightsquigarrow M$, where $\alpha$ is a constant action, and $N, M$ are states (see Figure 2). Figure 9 shows an Agda implementation of both the general definition of a context $\Gamma$ and one concrete $\Gamma_{1}$ that corresponds to the PDDL code snippet of Figure 1.

We now move on to our main goal: to realise the Curry-Howard intuition and define a framework in which plans will inhabit normalised formulae seen as types. We wish to show that, proving that a certain (possibly composite) plan $f$ satisfies pre- and postconditions given by the formulae $P$ and $Q$ will be equivalent to typing the judgement

$$
\Gamma \vdash f: P \downarrow_{+} \rightsquigarrow Q \downarrow_{+}
$$

We will say $P \downarrow_{+}$is the initial state of the plan $f$, and $Q \downarrow_{+}$is its final state. In the next section, we introduce typing rules that define derivations of these judgements. 


$$
\text { NilSub } \frac{}{\left[{ }^{1}<: N\right.} \text { ASub } \frac{N<: M \quad A^{t} \in M}{A^{t}, N<: M}
$$

Fig. 10. Sub-typing of normalised formulae.

$$
\text { Halt } \frac{M^{\prime}<: M}{\Gamma \vdash \text { halt }: M \rightsquigarrow M^{\prime}} \operatorname{Seq} \frac{\left(\alpha: M_{1}^{\prime} \rightsquigarrow M_{2}\right) \in \Gamma \quad \Gamma \vdash f: M_{1} \sqcup M_{2} \rightsquigarrow M_{3}}{\Gamma \vdash \alpha ; f: M_{1} \rightsquigarrow M_{3}}
$$

Fig. 11. Well-typing relation for plans.

$f: M \rightsquigarrow N$ can be typed given some planning domain $\Gamma$. A well-typed plan $\Gamma \vdash f$ : $M \rightsquigarrow N$ "transports" an initial state $M$ to a goal state $N$. The Agda code implements the typing relation as an inductive data type with two constructors, halt and seq, following verbatim Figure 11 (see also the accompanying Agda file). To exemplify these rules, we refer again to the Blocksworld problem with the pre-condition $P_{0}=$ onTable a $\wedge$

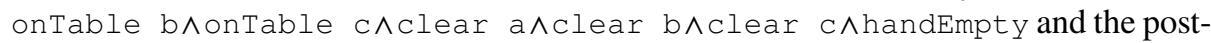
condition $Q_{0}=$ on a $\mathrm{b} \wedge$ on b c. Suppose that the PDDL planner proposes Plan1, as given in Section 2. Let plan 1 be the corresponding version in the precise mathematical notation of Figure 2 (cf. also its Agda version in Figure 8):

$$
\begin{gathered}
\operatorname{plan}_{1}=\text { pickup_from_table_b; putdown_on_stack_b_c; } \\
\text { pickup_from_table_a; putdown_on_stack_a_b; halt }
\end{gathered}
$$

If $\Gamma_{1} \vdash$ plan $_{1}: P_{0} \downarrow_{+} \rightsquigarrow Q_{0} \downarrow_{+}$yields a typing derivation by Figure 11 , then this typing derivation verifies that plan $_{1}$ correctly implements the given planning problem in the planning domain $\Gamma_{1}$ (cf. also Agda code for $\Gamma_{1}$ in Figure 9). To make our example more readable, we will use our mathematical notation. This gives the following definition of $\Gamma_{1}$, corresponding to the Agda code of Figure 9:

$$
\begin{aligned}
& \Gamma_{1}=\{\text { pickup_from_table_b : } \\
& \begin{array}{c}
\text { handEmpty } \wedge \\
\text { onTable } \mathrm{b} \wedge \\
\text { clear } \mathrm{b}
\end{array} \downarrow_{+} \rightsquigarrow \begin{array}{c}
\neg \text { handEmpty } \wedge \\
\text { holding } \mathrm{b}
\end{array} \downarrow_{+} \\
& \text {pickup_from_table_a : ... } \\
& \text { putdown_on_stack_b_c : } \\
& \neg \text { (holding b) } \wedge
\end{aligned}
$$

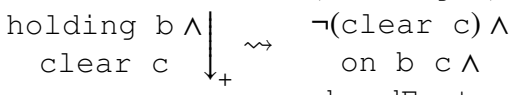

$$
\begin{aligned}
& \text { handEmpty } \downarrow_{+} \\
& \text {putdown_on_stack_a_b : ... }
\end{aligned}
$$

Let us perform the typing derivation for $\Gamma_{1} \vdash \operatorname{plan}_{1}: P_{0} \downarrow_{+} \rightsquigarrow Q_{0} \downarrow_{+}$. Given $P_{0} \downarrow_{+}$, then the first action that we can apply by the Seq rule is 


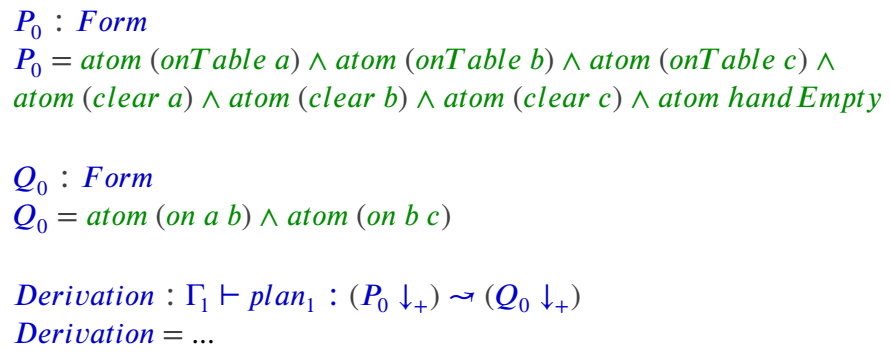

Fig. 12. Agda type-checking the derivation of $\Gamma_{1} \vdash$ plan $_{1}: P_{0} \downarrow_{+} \rightsquigarrow Q_{0} \downarrow_{+}$. We give the full code for Derivation in Appendix A or in [17].

pickup_from_table_b. The application of Seq demands that $P_{0} \downarrow_{+}$is a sub-type of the initial state of the action pickup_from_table_b in $\Gamma_{1}$. A sub-typing derivation provides such a proof, selecting the required piece of evidence from $P_{0} \downarrow_{+}$, i.e. handEmpty $\mathrm{y}^{+},(\text {onTable b) })^{+},(\text {clear b) })^{+}<$: $\quad(\text { onTable a })^{+},(\text {onTable b) })^{+}$, (onTable $\mathrm{c})^{+}$, (clear $\left.\mathrm{a}\right)^{+},(\text {clear } \mathrm{b})^{+},(\text {clear } \mathrm{c})^{+}$, handEmpty ${ }^{+}$. We have thus verified that plan $_{1}=$ pickup_from_table_b; $f^{\prime}$. To complete the proof of welltypedness and compute an action for $f^{\prime}$, we must show that the remainder of the plan is typeable. According to Seq, we now have a new state $P_{1}=P_{0} \downarrow_{+} \sqcup$ handEmpty ${ }^{-}$, ( onTable $\mathrm{b})^{-},(\text {holding } \mathrm{b})^{+}=(\text {onTable } \mathrm{a})^{+},(\text {onTable } \mathrm{b})^{-},(\text {onTable } \mathrm{c})^{+},($ clear $\mathrm{a})^{+},(\operatorname{clear} \mathrm{b})^{+},(\operatorname{clear} \mathrm{c})^{+},(\text {handEmpty })^{-},(\text {holding } \mathrm{b})^{+}$, as well as an obligation to prove $f^{\prime}: P_{1} \rightsquigarrow Q_{0} \downarrow_{+}$. We can pick the next action from $\Gamma_{1}$ : putdown_on_stack_b_c. Again $P_{1}$ is readily shown to be a sub-type of the preconditions of putdown_on_stack_b_c. Continuing in this way for each action in $\operatorname{plan}_{1}$, the final state is

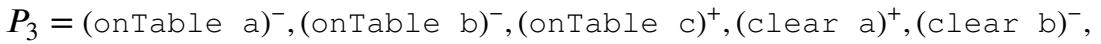

$$
\begin{aligned}
& \text { (clear } \left.\left.\left.\left.\mathrm{c})^{-} \text {, (on b c) }\right)^{+} \text {, handEmpty }{ }^{+} \text {, (holding b) }\right)^{-} \text {, (on a b }\right)^{+} \text {, (holding a }\right)^{-} \text {. }
\end{aligned}
$$

However, this is not the same state as the goal state $Q_{0}$. To resolve such cases, we have the rule Halt, eliminating all unnecessary evidence from the current state by proof of sub-typing i.e. $\Gamma_{1} \vdash$ halt $: P_{3} \rightsquigarrow Q_{0}$. Clearly (on a b) $)^{+}$, (on b c) $)^{+}<: P_{3}$ as required. We have thus verified that $\Gamma_{1} \vdash$ plan $_{1}: P_{0} \downarrow_{+} \rightsquigarrow Q_{0} \downarrow_{+}$. In Agda, the above derivation will amount to type-checking the function Derivation as shown in Figure 12. If it typechecks, then we know that plan ${ }_{1}$ can be soundly executed as a function. Proving this property in general is the subject of the next section.

\subsection{Computational Characterisation of Plans: Soundness of Plan Execution}

The proof of $\Gamma_{1} \vdash \operatorname{plan}_{1}: P_{0} \downarrow_{+} \rightsquigarrow Q_{0} \downarrow_{+}$provides evidence that the execution of plan 1 on a world satisfying $P_{0}$ produces a new world satisfying $Q_{0}$. Generally, the inference of $\Gamma \vdash f: M \rightsquigarrow N$, with $f=\alpha_{1} ; \ldots ; \alpha_{j}$; halt corresponds to successively applying actions $\alpha_{1} \ldots \alpha_{j}$ to states $M, M_{1}, \ldots M_{j}$ in a sequence of state transitions, satisfying $N<: M_{j}$. We now prove that the plan $f$ thus inferred indeed has a computational 


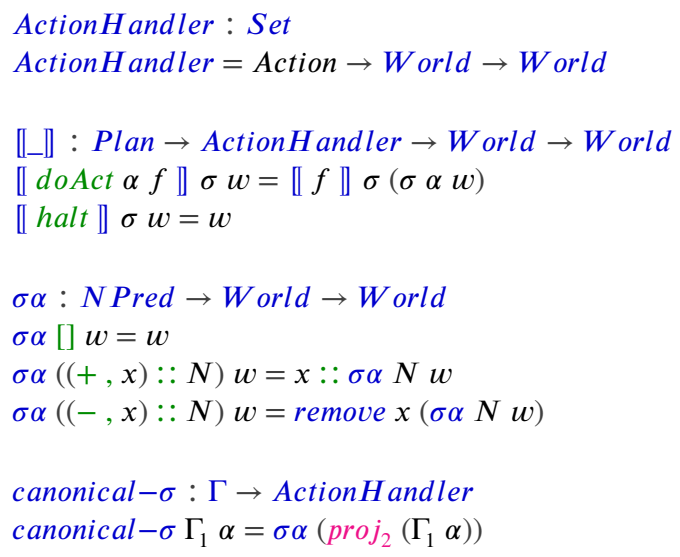

Fig. 13. Agda code for an action handler, an evaluation function and a canonical handler.

meaning, i.e. can be evaluated, and that the result of its evaluation is sound. To state this, we need to define an evaluation function $\llbracket . \rrbracket$ that will interpret actions on worlds. Recall that every state $N$ maps to a world $w_{N}$. Let us use notation $\sigma$ for an arbitrary mapping (an action handler) that maps each action $\alpha: M \rightsquigarrow N$ to insertions and deletions on the world $w_{M}$ according to $\alpha$ 's action on $M$. We then define the evaluation function $\llbracket \rrbracket \frac{\sigma}{w}$ that evaluates a plan to a world (according to given world $w$ and action handler $\sigma$ ):

$$
\begin{aligned}
\llbracket \text { halt } \rrbracket_{w}^{\sigma} & =w \\
\llbracket \alpha ; f \rrbracket_{w}^{\sigma} & =\llbracket f \rrbracket_{(\sigma \alpha w)}^{\sigma}
\end{aligned}
$$

We say that an action handler $\sigma$ is well-formed if, for any $w \in\left\{w_{M}\right\}, M^{\prime}<: M$ and $\alpha: M^{\prime} \rightsquigarrow N$ in $\Gamma$ it follows that $(\sigma \alpha w) \in\left\{w_{M \sqcup N}\right\}$. Figure 13 shows Agda definitions of an action handler and evaluation action.

Canonical Handler. In order to be constructive in our further claims, and to provide a practical solution to the quest for a well-formed handler, we first define a canonical handler for a given context (planning domain). Firstly, we define a function $\sigma_{\alpha}$ that constructs a world from a state:

$$
\begin{aligned}
\sigma_{\alpha}[] w & =w \\
\sigma_{\alpha}\left[A^{+}, N^{\prime}\right] w & =\sigma_{\alpha} N^{\prime}(w \cup\{A\}) \\
\sigma_{\alpha}\left[A^{-}, N^{\prime}\right] w & =\sigma_{\alpha} N^{\prime}(w \backslash\{A\})
\end{aligned}
$$

Next, given a context $\Gamma$, we apply $\sigma_{\alpha}$ to $N$ for each $\alpha: M \rightsquigarrow N$ in $\Gamma$; thus obtaining a canonical mapping $\sigma_{\alpha} \Gamma$ from actions and worlds into worlds, as required. The resulting canonical action handler is well-formed, as long as the states to which it is applied are consistent, in the following sense: 
Implicit consistency assumption: for every state $N$, if $A^{t} \in N$ then $A^{-t} \notin N$.

Proposition 1. Given a context $\Gamma$, the canonical action handler $\sigma_{\alpha} \Gamma$ is well-formed.

Proof. The proof starts with considering an arbitrary state $M$ with $w \in\left\{w_{M}\right\}$, an arbitrary $A^{t} \in M$, and an arbitrary action $\alpha$ in $\Gamma$ such that $M^{\prime}<: M$ and $\alpha: M^{\prime} \rightsquigarrow N$. It proceeds by considering two cases, when $t=+$ and $t=-$, and consequently when $A \in w$ or $A \notin w$. In each of these cases, it considers all possible effects of $\sigma_{\alpha}$ (i.e. formula deletions and insertions) in the process of constructing the world $w^{\prime}=$ canonical $-\sigma \alpha w$. The attached Agda file gives the full proof. It uses the implicit consistency assumption to eliminate the cases when states are inconsistent and hence when more than one choice for deletion/insertion are possible.

The next two theorems show that executing a well-typed plan $f$ by the evaluation function $\llbracket f \rrbracket_{w}^{\sigma}$ is sound, for any well-formed handler $\sigma$.

Theorem 2 (Soundness of evaluation for normalized formulae). Suppose $\Gamma \vdash f$ : $M \rightsquigarrow N$. Then for any $w \in\left\{w_{M}\right\}$, and any well-formed handler $\sigma$, it follows that $\llbracket f \rrbracket_{w}^{\sigma} \in\left\{w_{N}\right\}$.

Proof. The proof proceeds by structural induction on the typing derivation $\Gamma \vdash f$ : $M \rightsquigarrow N$.

Case 1 (Halt). By assumption $w \in\left\{w_{M}\right\}$ and thus because $N<$ : $M$, it follows $w \in$ $\left\{w_{N}\right\}$. Since $\llbracket$ halt $\rrbracket_{w}^{\sigma}=w$, we get $\llbracket$ halt $\rrbracket_{w}^{\sigma} \in\left\{w_{N}\right\}$ as required.

Case 2 (Seq). Note that $f=\alpha ; f^{\prime}$ and therefore $\alpha: M^{\prime} \rightsquigarrow M_{2}$ is in $\Gamma$ and $\Gamma \vdash$ $f^{\prime}: M \sqcup M_{2} \rightsquigarrow N$ by inversion on $\Gamma \vdash \alpha ; f^{\prime}: M \rightsquigarrow N$. Then by induction every $w^{\prime} \in\left\{w_{M \sqcup M_{2}}\right\}$ gives $\left(\llbracket f \rrbracket_{w^{\prime}}^{\sigma}\right) \in\left\{w_{N}\right\}$ for any well-formed $\sigma$. However, by the well-formedness of $\sigma$ and because $w \in\left\{w_{M}\right\}$, we have $(\sigma \alpha w) \in\left\{w_{M \sqcup M_{2}}\right\}$. Thus $\llbracket f^{\prime} \rrbracket_{(\sigma \alpha \omega)}^{\sigma} \in\left\{w_{N}\right\}$ and therefore $\llbracket f \rrbracket_{w}^{\sigma} \in\left\{w_{N}\right\}$.

Theorem 3 (Soundness of evaluation). Suppose $\Gamma \vdash f: P \downarrow_{+} \rightsquigarrow Q \downarrow_{+}$then for any $w$ such that $w \vDash_{+} P$, and any well-formed $\sigma$ it follows $\llbracket f \rrbracket_{w}^{\sigma} \vDash_{+} Q$.

Proof. By assumption $w k_{+} P$ and by the completeness of normalisation (Theorem 1), we have $w \in\left\{w_{P \downarrow_{+}}\right\}$. Then from Theorem 2, we have $\llbracket f \rrbracket_{w}^{\sigma} \in\left\{w_{Q \downarrow_{+}}\right\}$. Thus by the soundness of normalisation (Theorem 1), obtain $\llbracket f \rrbracket_{w}^{\sigma} \vDash_{+} Q$.

Thus the derivation of a type for a plan $f$ induces a proof that the execution of a plan in world $w$ is correct. Although neither of the above theorems depends on the implicit consistency assumption for its proofs, the existence of a well-formed and canonical handler is predicated upon the consistency assumption. Our Agda implementation of a canonical handler (cf. Figure 13) allows us to fully harness the computational properties of plans. For the Blocksworld example, we can directly evaluate $\llbracket$ plan $_{1} \rrbracket_{w}^{\sigma}$ by plugging in:

- in place of $w$ - the world resulting from computing $\sigma_{\alpha}\left(P_{0} \downarrow_{+}[]\right) \varnothing$. (To see this, recall that $P_{0}$ is the formula that described the initial state in all examples of the previous section, and $P_{0} \downarrow_{+}$[] is the state resulting from normalising $P_{0}$.) 
- and in place of $\sigma$ - the canonical handler for $\Gamma_{1}$. (Recall that $\Gamma_{1}$ is the context that defined the given planning domain in the previous section.)

In Agda, we simply evaluate the term:

$$
\llbracket \text { plan }_{1} \rrbracket\left(\text { canonical }-\sigma \Gamma_{1}\right)\left(\sigma \alpha\left(P_{0} \downarrow[+][]\right)[]\right)
$$

Evaluation of this term results, just as we manually computed in the last section, in a world $w^{\prime}=\{$ handEmpty, on a b, on b c, clear a, onTable c (in Agda syntax: hand Empty $::($ on $a b)::($ on $b c)::$ (clear a) $::($ onTable $c)::$ []). That is, the world that corresponds to the state $P_{3}$ of the previous section. Observe that $w^{\prime}=\sigma_{\alpha} P_{3} \varnothing$.

\section{Discussion, Conclusions, and Future Work}

We have given a proof of concept formalisation of a subset of PDDL plans in type theory. In line with the Curry-Howard approach to first-order logic, we formulated an inference system that treats planning domains as types, and generated plans as functions that inhabit these types. Type-checking then ensures the soundness of these executable functions relative to the specifications given as types. This paper does not cover the whole PDDL syntax, nor does it implement the search and decision procedures of a usual automated planner e.g. the Stanford Research Institute Problem Solver STRIPS [4]. Rather, our contribution is in setting up the original design of a method of Curry-Howard approach to AI planning languages in general, as well as showing the feasibility of its successful implementation in a dependently typed language, such as Agda. This dual purpose has determined our style of presentation, in which the formal method has been given in parallel with, but independently from, the Agda code.

Further experiments with plans: In the accompanying implementation [17], we provide a second fully implemented example of a PDDL domain and plan checking in Agda: for a Logistic planning problem. The problem consists of finding the best route (airplanes, tracks, cities of call) to deliver a given parcel to a given office. The experiment showed that, once the main Agda implementation is set up, instantaiting it with various problems only takes a routine boilerplate code (such as e.g. proofs of decidability of equality on predicates). Generation of this boilerplate code can in future be fully automated using code reflection. Throughout our implementation, we have been working with plans generated by an on-line PDDL editor http: //editor.planning.domains/. In the future, a parser can be added to convert PDDL syntax directly into Agda.

Computational content of plans and the implicit consistency assumption: As Proposition 1 has shown, the existence of a canonical and well-formed handler depends crucially on the implicit consistency assumption. At the same time, the proofs of Theorems 2 or 3 do not depend on the consistency assumption. Thus, as we show in Appendix B, it is possible in principle to construct planning domains and problems that violate the assumption but are accepted by the well-typedness relation of Figure 11. However, if such examples are added to the system, the implicit consistency assumption needs to be removed (or else $\perp$ will become provable, as Appendix B shows). But without the 
assumption, we lose the existence of a well-formed and canonical handler and thus the ability to evaluate the plans. This situation is of course illustrative of the rigour and transparency that a constructive approach brings to verification. In our case, it dictates that any practical deployment of the presented prototype needs to enforce the consistency assumption. This can be done by either embedding additional state consistency checks or by implementing states as partial functions from formulae to Booleans.

Related work: Verification of AI languages and applications is an active research field. In planning languages, two major trends exist. Firstly, PDDL is used to verify autonomous systems and applications, see e.g. [14]; and it has been successfully integrated within other similar languages, such as GOLOG [10], with the purpose of verifying plans written in the situation calculus [1,21]. Secondly, planning domains have been verified using model checkers [9], other automated provers such as Event-B [5], or planning support tools such as VAL [8]. The method that we have presented is complementary to these two trends. Its main difference lies in taking the perspective of intrinsic, rather than external verification. That is, the correctness of the generated plans is verified not by an external tool, such as a model checker, but is performed intrinsically within the code that implements the plans. At the same time, the code that implements the plans is inseparable from the language in which planning domains are specified. To our knowledge, this is the first attempt to bring these benefits of the Curry-Howard approach to automated planning languages. Provisioning types for plans not only equips planners with certificates of correctness for inspection, but also provides a direct link to an implementation's type theory. This provides a convenient path to compiler verification.

Current limitations and possible improvements: There are a number of obvious improvements that could be made to our current work.

First-order planning domains and first-order types: Although the technical development of the code that we have presented takes full advantage of Agda's dependent types, the types that represent the predicates and formulae of the planning problems are given by simple types. This is because we propositionalised the planning domains. We however hope to extend this initial framework to the full first-order syntax of PDDL. This development will also involve the following extensions.

Beyond consistency assumptions; constraints: We have discussed the implicit consistency assumption that our approach imposes. More generally, we note that PDDL lacks any general method of handling consistency as well as similar but more complex constraints and invariants, such as, for example, constraints saying that handEmpty and holding $x$ are mutually exclusive. This is a complex problem, but one for which we anticipate that our dependently-typed setting will soon provide some useful solutions.

Functions and higher-order plans: The design of this Agda prototype has revealed several limitations in state-of-the-art implementations of planning languages: e.g. their reliance on the closed word assumption and formulae grounding, the absence of functions, and the restricted use of disjunctions. Again, we see a potential of our method to overcome many of these limitations thanks to our general dependently-typed set-up, in which the use of functions, higher-order features, constraints and effect handling will be much more natural than in the current implementations. 


\section{References}

1. Claßen, J., Eyerich, P., Lakemeyer, G., Nebel, B.: Towards an integration of golog and planning. In: Veloso, M.M. (ed.) IJCAI 2007, Proceedings of the 20th International Joint Conference on Artificial Intelligence, Hyderabad, India, January 6-12, 2007. pp. 1846-1851 (2007)

2. De Moura, L., Bjørner, N.: Z3: An efficient SMT solver. In: Proceedings of the Theory and Practice of Software, 14th International Conference on Tools and Algorithms for the Construction and Analysis of Systems. pp. 337-340. TACAS'08/ETAPS'08, Springer-Verlag, Berlin, Heidelberg (2008)

3. Dutertre, B., De Moura, L.: The Yices SMT solver. Tech. rep. (8 2006), tool paper at http://yices.csl.sri.com/tool-paper.pdf

4. Fikes, R., Nilsson, N.J.: STRIPS: A new approach to the application of theorem proving to problem solving. Artificial Intelligence 2(3/4), 189-208 (1971)

5. Fourati, F., Bhiri, M.T., Robbana, R.: Verification and validation of PDDL descriptions using Event-B formal method. In: 2016 5th International Conference on Multimedia Computing and Systems (ICMCS). pp. 770-776 (Sept 2016)

6. Fu, P., Komendantskaya, E.: Operational semantics of resolution and productivity in horn clause logic. Formal Asp. Comput. 29(3), 453-474 (2017)

7. Fu, P., Komendantskaya, E., Schrijvers, T., Pond, A.: Proof relevant corecursive resolution. In: Kiselyov, O., King, A. (eds.) Functional and Logic Programming - 13th International Symposium, FLOPS 2016, Kochi, Japan, March 4-6, 2016, Proceedings. Lecture Notes in Computer Science, vol. 9613, pp. 126-143. Springer (2016)

8. Howey, R., Long, D., Fox, M.: VAL: Automatic plan validation, continuous effects and mixed initiative planning using PDDL. In: 16th IEEE International Conference on Tools with Artificial Intelligence. pp. 294-301 (2004)

9. Khatib, L., Muscettola, N., Havelund, K.: Verification of plan models using UPPAAL. In: Rash, J.L., Rouff, C.A., Truszkowski, W., Gordon, D.F., Hinchey, M.G. (eds.) Formal Approaches to Agent-Based Systems, First International Workshop, FAABS 2000 Greenbelt, MD, USA, April 5-7, 2000, Revised Papers. Lecture Notes in Computer Science, vol. 1871, pp. 114-122. Springer (2000)

10. Levesque, H.J., Reiter, R., Lespérance, Y., Lin, F., Scherl, R.B.: GOLOG: A logic programming language for dynamic domains. The Journal of Logic Programming 31(1), 59-83 (1997)

11. McDermott, D., Ghallab, M., Howe, A., Knoblock, C., Ram, A., Veloso, M., Weld, D., Wilkins, D.: PDDL - The Planning Domain Definition Language (Version 1. 2). Technical Report CVC TR-98-003/DCS TR-1165, Yale Center for Computational Vision and Control (1998)

12. Necula, G.C.: Proof-carrying code. In: POPL. pp. 106-119 (1997)

13. Ong, L.: Higher-order model checking: An overview. In: 30th Annual ACM/IEEE Symposium on Logic in Computer Science, LICS 2015, Kyoto, Japan, July 6-10, 2015. pp. 1-15 (2015)

14. Raimondi, F., Pecheur, C., Brat, G.: PDVer, a tool to verify PDDL planning domains. In: ICAPS'09 Workshop on Verification and Validation of Planning and Scheduling Systems, September 20, 2009, Thessaloniki, Greece (2009)

15. Reiter, R.: On closed world data bases. In: Gallaire, H., Minker, J. (eds.) Logic and Data Bases, pp. 55-76. Plenum Press, New York (1978)

16. Schubert, A., Urzyczyn, P.: Answer set programming in intuitionistic logic. Indagationes Mathematicae 29(1), 276 - 292 (2018), 1.E.J. Brouwer, fifty years later

17. Scwaab, C., Hill, A., Farka, F., Komendantskaya, E.: Proof-carrying plans: Agda implementation and examples (2018), https: / / github.com/PDTypes 
18. Sorensen, M.H., Urzyczyn, P.: Lectures on the Curry-Howard Isomorphism, Studies in Logic, vol. 149 (2006)

19. Wadler, P.: Propositions as types. Commun. ACM 58(12), 75-84 (2015)

20. Waldinger, R.J.: Achieving several goals simultaneously. Machine Intelligence 8 (1977)

21. Zarrieß, B., Claßen, J.: Decidable verification of GOLOG programs over non-local effect actions. In: Schuurmans, D., Wellman, M.P. (eds.) Proceedings of the Thirtieth AAAI Conference on Artificial Intelligence, February 12-17, 2016, Phoenix, Arizona, USA. pp. 11091115. AAAI Press (2016) 


\section{A The full code for Derivation of Figure 12}

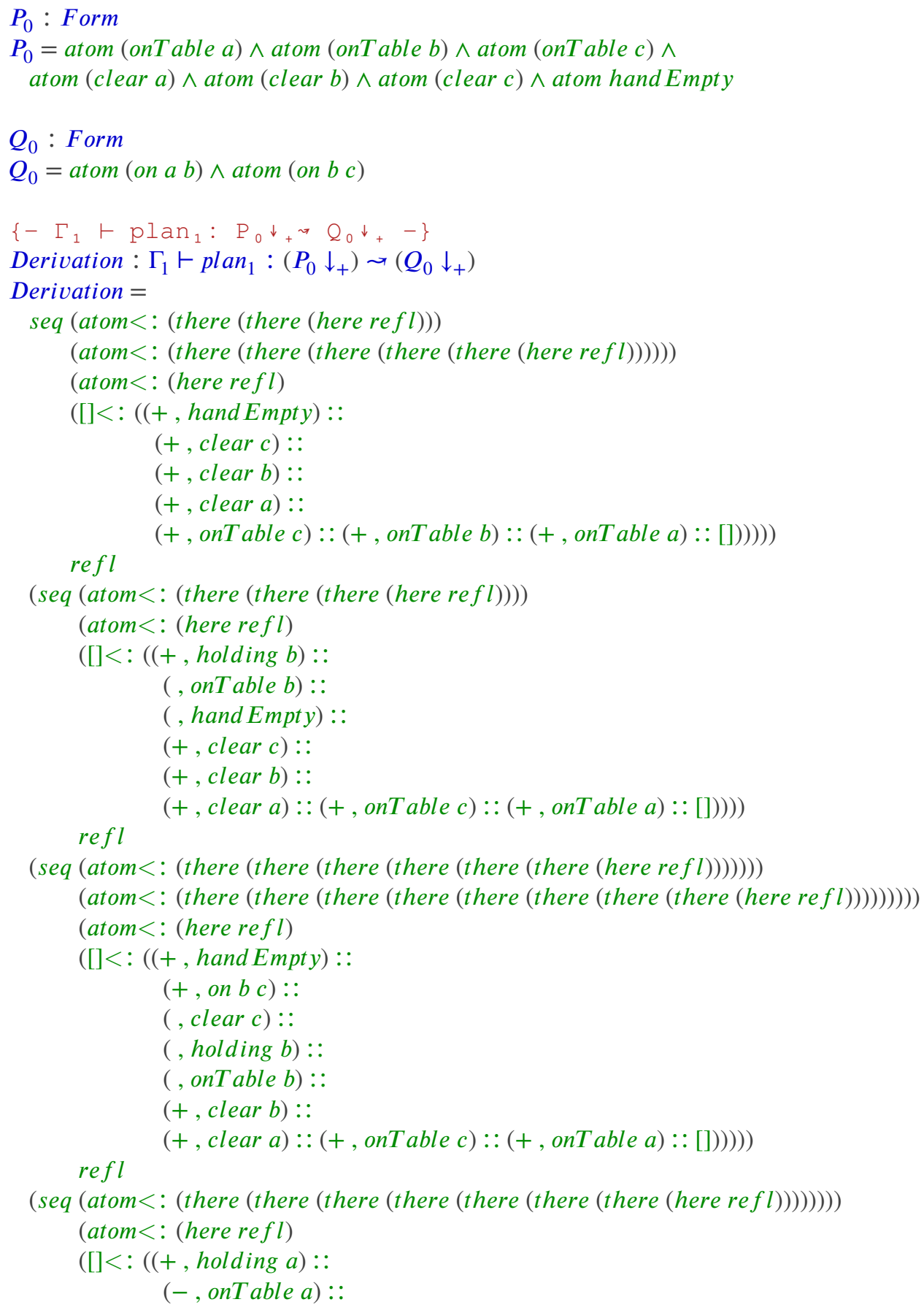




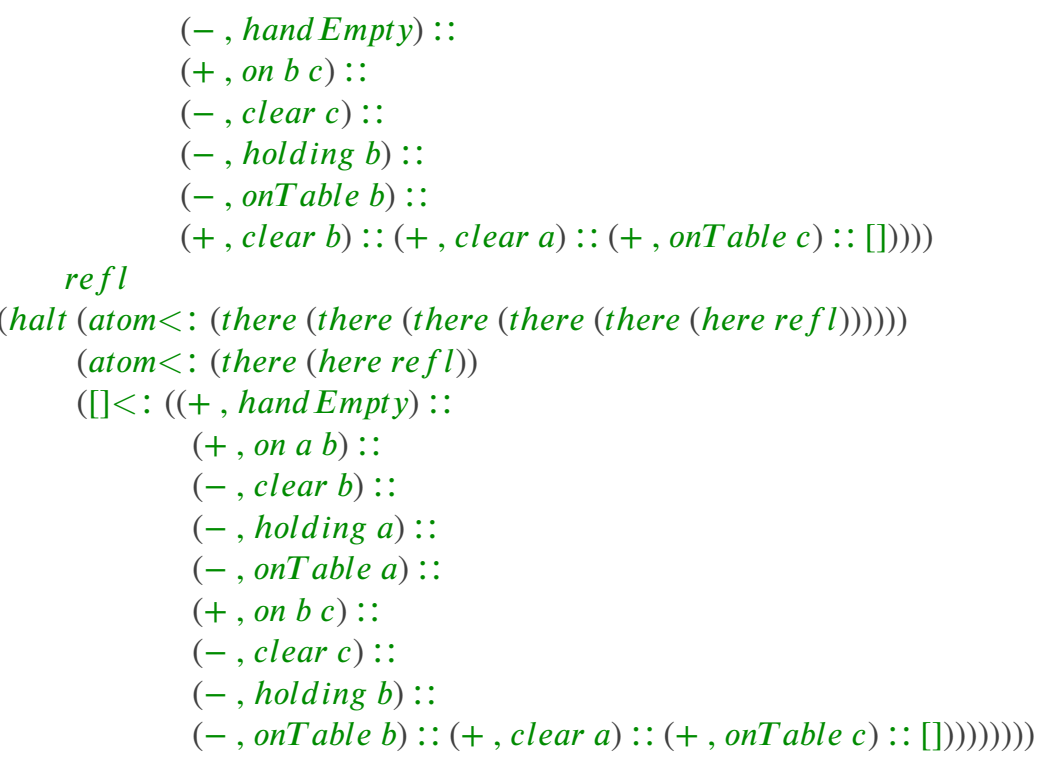




\section{B Experiment with Inconsistent States}

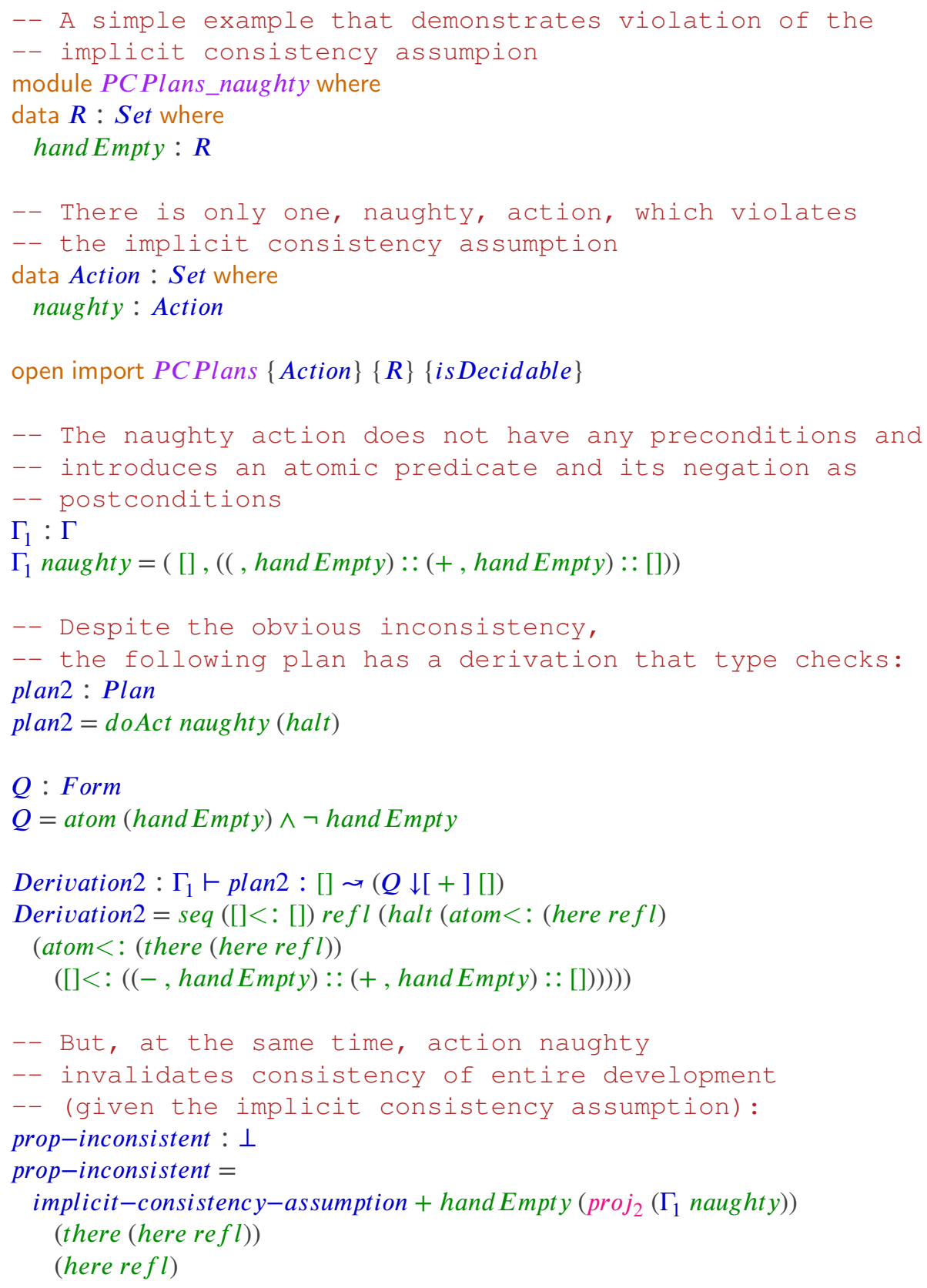

